\title{
ON LISKOVETS APPROACH FOR NONLINEAR ILL-POSED PROBLEMS UNDER ARBITRARILY PERTURBATIVE OPERATORS ${ }^{1}$
}

\author{
NGUYEN BUONG
}

\begin{abstract}
The purpose of this paper is to present a modification of Liskovets's approach of solution of nonlinear ill-posed problems involving monotone operators in real reflexive Banach space under arbitrarily perturbative operators. The aspect of convergence of Tikhonov regularization is considered in combination with finite-dimensional approximations of the space.
\end{abstract}

\section{INTRODUCTION}

Let $X$ be a real reflexive Banach space having property: $X$ and $X^{*}$ are strictly convex and weak convergence and convergence of norms of any sequence in $X$ follow its strong convergence, where $X^{*}$ denotes the dual space of $X$. For the sake of simplicity norms of $X$ and $X^{*}$ will be denoted by one symbol $\|\cdot\|$. We write $\left\langle x^{*}, x\right\rangle$ instead of $x^{*}(x)$ for $x^{*} \in X^{*}$ and $x \in X$. Let $A$ be a monotone, continuous and bounded operator with domain $D(A)=X$ and range $R(A) \subseteq X^{*}$.

Many problems arising not only in mathematical analysis but also in practice (see $[6,10,12])$ can be written in the form of operator equation of the first kind

$$
A(x)=f_{0}, f_{0} \in R(A) .
$$

Without additional conditions on the structure of $A$, as strongly or uniformly monotone property, this equations is one of ill-posed problems. By this we mean that the solutions of (1.1) do not depend continuously on the data $\left(A, f_{0}\right)$. To solve it we have to use stable methods. A widely used and effective method is Tikhonov regularization that consists of minimizing some functional depending on a small parameter (see [11]). For the class of problem involving monotone operators there exists another more convenient version of Tikhonov regularization in form of operator equation

$$
A_{h}(x)+\alpha U^{s}(x)=f_{\delta},
$$

where $\left(A_{h}, f_{\delta}\right)$ are approximations for $\left(A, f_{0}\right)$ such that $A_{h}$ are monotone,

$$
\left\|A_{h}(x)-A(x)\right\| \leq h g(\|x\|), \forall x \in X,\left\|f_{\delta}-f_{0}\right\| \leq \delta,
$$

with the well known levels $(\delta, h) \rightarrow 0$ and bounded, continuous and nondecreasing function $g(t)$; the parameter $\alpha$ is called the parameter of regularization and $U^{*}$ is the dual mapping of $X$ satisfying the condition

$$
\left\langle U^{s}(x), x\right\rangle=\|x\|^{s},\left\|U^{s}(x)\right\|=\|x\|^{s-1}, s \geq 2 .
$$

In the case of Hilbert space $U^{s}(x)=I, I$ denotes the identity operator, the algorithm (1.2) was studied in [1]. If $s=2$, it was investigated in $[3,8]$. If $s$ is an arbitrary number such

\footnotetext{
${ }^{1}$ Supported by the State Program for Fundamental Researches in Natural Sciences
} 
algorithm was studied in $[2,4]$ under the conditions

$$
\left\langle U^{s}(x)-U^{s}(y), x-y\right\rangle \geq m\|x-y\|^{s}, \quad m>0,
$$

If $A_{h}$ are not monotone, Equation (1.2) does not always have solution. So in [7] O.A. Liskovets constructed regularized solutions $x^{\omega}$ which are the solutions of the following variational inequalities

$$
\left\langle A_{h}\left(x^{\omega}\right)+\alpha U^{v}\left(x^{\omega}\right)-f_{\delta}, x-x^{\omega}\right\rangle \geq-\varepsilon g\left(\left\|x^{\omega}\right\|\right) \| x-x^{\omega}, \forall x \in X, \varepsilon \geq h, \alpha>0
$$

under the additional condition

$$
0 \leq g(t) \leq M_{1}+N_{1} t, M_{1}, N_{1}>0 .
$$

It is sill open the question about the case when $g(t)$ does not possess the last property, i.e. $g(t)$ increases faster than $t$, as $t \rightarrow+\infty$. In this note, we answer this question in the case of Banach space $X$. More precisely, in Section 2, we present a modification of Liskovets approach and consider it in combination with finite-dimensional approximations of the Banach space $X$.

Below, the symbols $\rightarrow$ and $\rightarrow$ denote weak convergence and convergence in norm, respectively.

\section{MAIN RESULTS}

Let there exists a convex, closed and bounded subset $G$ of $X$ such that $\|x\| \leq M, x \in G$ and Int $S_{0}^{G} \neq \emptyset$, where $S_{0}^{G}:=S_{0} \cap G, S_{0}$ is the set of solution of (1.1) and $M$ is a positive constant.

Instead of (1.4) we consider the following inequality

$$
\left\langle A_{h}(x)+\alpha U^{s}(x)-f_{\delta}, x-x_{\varepsilon}\right\rangle+(g(M) \varepsilon+\delta)\left\|x-x_{\epsilon}\right\| \geq 0, x_{\omega} \in G, \forall x \in G, \alpha>0, \varepsilon \geq h .
$$

Lemma 2.1. For every fixed $\alpha>0, \varepsilon \geq h$ and $\delta$ the set $S_{\omega}$ of solutions of (2.1) is nonempty convex and closed.

Proof. Let $x_{\alpha} \in G$ be a unique solution of the following variational inequality (see [9])

$$
\left\langle A(x)+\alpha U^{s}(x)-f_{0}, x-x_{\alpha}\right\rangle \geq 0, \forall x \in G .
$$

Then

$$
\begin{gathered}
\left\langle A_{h}(x)+\alpha U^{s}(x)-f_{\delta}, x-x_{\alpha}\right\rangle+(g(M) \varepsilon+\delta)\left\|x-x_{\alpha}\right\|=\left\langle A_{h}(x)-A(x), x-x_{\alpha}\right\rangle \\
+\left\langle A(x)+\alpha U^{s}(x)-f_{0}, x-x_{0}\right\rangle+\left\langle f_{0}-f_{\delta}, x-x_{\alpha}\right\rangle+(g(M) \varepsilon+\delta)\left\|x-x_{\alpha}\right\| \\
\geq(g(M) \varepsilon+\delta)\left\|x-x_{\alpha}\right\|-(g(M) h+\delta)\left\|x-x_{\alpha}\right\| \geq 0 .
\end{gathered}
$$

i.e., $S_{\omega} \neq \emptyset$. The closed and convex property is verified by using (2.1).

Theorem 2.2. The sequence $\left\{x_{\omega}\right\}$ converges to $x_{0}^{G} \in S_{0}^{G}:\left\|x_{0}^{G}\right\|=\min \left\{\|x\|, x \in S_{0}^{G}\right\}$, as $\varepsilon / \alpha, \delta / \alpha$ and $\alpha$ tend to zero, where $x_{\omega} \in S_{\omega}$ is chosen arbitrarily for every fixed $\alpha>0$.

Proof. Indeed, since $G$ is bounded the sequence $\left\{x_{\omega}\right\}$ is bounded, too. Let $x_{\omega} \rightarrow \tilde{x}$. Then $\tilde{x} \in G$, because each convex and closed set in $X$ also is weakly closed in $X$. On the other hand, from (2.1) we have 


$$
\left\langle A(x)-f_{\delta}, x-x_{\omega}\right\rangle+\alpha\left\langle U^{s}(x), x-x_{\alpha}\right\rangle+2 M(g(M) \varepsilon+\delta)\left\|x-x_{\omega}\right\| \geq 0, \forall x \in G .
$$

After passing $\varepsilon, \delta$ and $\alpha$ to zero in this inequality we have got

$$
\left\langle A(x)-f_{0}, x-\tilde{x}\right\rangle \geq 0, \forall x \in G .
$$

If $\tilde{x} \in \operatorname{Int} G$, then $A(\tilde{x})=f$ by Minty's lemma (see [13]). Let

$$
G^{0}=\left\{x^{1} \in G:\left\langle A(x)-f_{0}, x-x^{1}\right\rangle \geq 0, \forall x \in G\right\} .
$$

Evidently, $S_{0}^{G} \subset G^{0}$. Let $\tilde{x} \in G^{0}$, but $\tilde{x} \notin S_{0}^{G}$. If $\tilde{x} \in \operatorname{Int} G^{0}$, then $\tilde{x} \in S_{0}^{G}$. It means that $\tilde{x} \in \operatorname{Fr} G^{0}$, the frontier of $G$. It is impossible, since the sets $G^{0}$ and $S_{0}^{G}$ are both closed and Int $G^{0}=\operatorname{Int} S_{0}^{G}$. Consequently, from (2.2) we have $\tilde{x} \in S_{0}^{G}$.

Replacing $x$ by $t x+(1-t) x_{\omega}, t \in(0,1)$ in $(2.1$ and after dividing both hand sides of the obtained inequality by $t$ and then tending $t$ to zero we get

$$
\left\langle A_{h}\left(x_{\omega}\right)+\alpha U^{s}\left(x_{\omega}\right)-f_{\delta}, x-x_{\omega}\right\rangle+(g(M) \varepsilon+\delta)\left\|x-x_{\omega}\right\| \geq 0, \forall x \in G .
$$

Therefore, using (1.3) we obtain

$$
m\left\|x-x_{\omega}\right\|^{s} \leq\left\langle U^{s}(x), x-x_{\omega}\right\rangle+2(g(M) \varepsilon+\delta)\left\|x-x_{\omega}\right\| / \alpha, \forall x \in S_{0}^{G} .
$$

From this inequality it implies that the sequence $\left\{x_{\omega}\right\}$ converges strongly to $\tilde{x}$ and

$$
\left\langle U^{s}(x), x-\tilde{x}\right\rangle \geq 0, \forall x \in S_{0}^{G} .
$$

We shall prove that $\tilde{x}=x_{0}^{G}$. Since $S_{0}^{G}$ is convex and $U^{s}$ is hemicontinuous (because of strictly convex property of $X^{*}$ see [11]) the last inequality is equivalent to

$$
\left\langle U^{s}(\tilde{x}), x-\tilde{x}\right\rangle \geq 0, \forall x \in S_{0}^{G} .
$$

Hence $\|\tilde{x}\| \leq\|x\|, \forall x \in S_{0}^{G}$. Because of the strictly convex property of $X \tilde{x}=x_{0}^{G}$.

Now, we can approximate the inequality (2.1) by the sequence of finite-dimensional problems

$$
\begin{gathered}
\left\langle A_{h}^{n}\left(x^{n}\right)+\alpha U^{s n}\left(x^{n}\right)-f_{\delta}^{n}, x^{n}-x_{\omega n}\right\rangle+(g(M) \varepsilon+\delta)\left\|x^{n}-x_{\omega n}\right\| \geq 0, \\
x_{\omega n} \in G_{n}, \forall x^{n} \in G_{n}=P_{n} G, x^{n}=P_{n} x, x \in G .
\end{gathered}
$$

where $A_{h}^{n}=P_{n}^{*} A_{h} P_{n}, U_{n}^{s}=P_{n}^{*} U^{s} P_{n}, f_{\delta}^{n}=P_{n}^{*} f_{\delta}$ and $P_{n}$ denotes the linear projection form $X$ on its subspace $X_{n}$ satisfying the condition

$$
X_{n} \subset X_{n+1}, P_{n} x \rightarrow x, n \rightarrow+\infty, \forall x \in X
$$

and $P_{n}^{*}$ is the adjoint of $P_{n},\left\|P_{n}\right\| \leq C, C$ is the positive constant $(C \geq 1)$. The existence of solution $x_{\omega n}$ of (2.3) is proved by the similar way as for (2.1).

We establish whether

$$
\lim _{\substack{\alpha, h, \delta \rightarrow 0 \\ n \rightarrow+\infty}} x_{\omega n}=x_{0}^{G} .
$$

Obviously, the answer for this question depends on the relation between $h, \alpha, \delta$ and $n$. Applying the idea of W. Engl and C. Groetsch in [5] we are going to answer this question. 
Theorem 2.3. Assume that following conditions hold:

(i) $A$ is Fréchet differentiable in some neighbourhood $O_{0}$ of $S_{0} s-1$-times if $s=[s]$, the integer part of $s,[s]$ - times of $s \neq[s]$.

(ii) There exists a constant $\tilde{L}>0$ such that

$$
\left\|A^{(k)}(x)=A^{(k)}(y)\right\| \leq \tilde{L}\|x-y\|, \forall x \in S_{0}, y \in 0,
$$

$k=s-1$ if $s=[s], k=[s]$ if $s \neq[s]$, and if $[s] \geq 3$, then $A^{(2)}(x)=\cdots=A^{(k)}(x)=0$.

(iii) $\alpha=\alpha(n) \rightarrow 0$ such that

$$
\left(\gamma_{n}(x)+\left\|\left(I-P_{n}\right) x\right\|^{[s]}\right) \alpha^{-1} \rightarrow 0, \forall x \in S_{0},
$$

as $n \rightarrow+\infty$, where $\gamma_{n}(x)$ is defined by $\gamma_{n}(x)=\left\|A^{\prime}(x)\left(I-P_{n}\right) x\right\|$.

Then the sequence $\left\{x_{\omega n}\right\}$ converges to $x_{0}^{G}$.

Proof. As in the proof of Theorem 2.2, from (2.3) we can obtain the inequality

$$
\left\langle\dot{A}_{h}^{n}\left(x_{\omega n}\right)+\alpha U^{s n}\left(x_{\omega n}\right)-f_{\delta}^{n}, x^{n}-x_{\omega n}\right\rangle+(g(M) \varepsilon+\delta)\left\|x^{n}-x_{\omega n}\right\| \geq 0, \forall x^{n} \in G_{n} .
$$

Thus

$$
\begin{aligned}
\alpha m\left\|x_{\omega n}-x^{n}\right\|^{s} & \leq(g(M) \varepsilon+\delta)\left\|x_{\omega n}-x^{n}\right\|+\alpha\left\langle U^{s}\left(x^{n}\right), x^{n}-x_{\omega n}\right\rangle \\
& +\left\langle A_{h}\left(x_{\omega n}\right)-A\left(x_{\omega n}\right)-A\left(x^{n}\right)+A\left(x^{n}\right)-A(x)+f_{0}-f_{\delta}, x^{n}-x_{\omega n}\right\rangle, \\
& x \in S_{0}^{G}, x^{n}=P_{n} x .
\end{aligned}
$$

Since

$$
\begin{gathered}
\left\|A_{h}\left(x_{\omega n}\right)-A\left(x_{\omega n}\right)\right\| \leq h g\left(\left\|x_{\omega n}\right\|\right), \\
\left\langle A\left(x_{\omega n}\right)-A\left(x^{n}\right), x^{n}-x_{\omega n}\right\rangle \leq 0,\left\|f_{0}-f_{\delta}\right\| \leq \delta,
\end{gathered}
$$

we obtain

$$
\alpha m\left\|x_{\omega n}-x^{n}\right\|^{s} \leq\left(2(g(M) \varepsilon+\delta)+\left\|A\left(x^{n}\right)-A(x)\right\|\right)\left\|x_{\omega n}-x^{n}\right\|+\alpha\left\langle U^{s}\left(x^{n}\right), x^{n}-x_{\omega n}\right\rangle .
$$

If $s=[s]$, we can write

$$
A\left(x^{n}\right)=A(x)+A^{\prime}(x)\left(x^{n}-x\right)+r_{n}
$$

with

$$
\left\|r_{n}\right\| \leq \frac{L}{s !}\left\|\left(I-P_{n}\right) x\right\|^{s} .
$$

Therefore, from (2.4) it is easy to see that

$$
\begin{aligned}
\alpha m\left\|x_{\omega n}-x^{n}\right\|^{s} & \leq\left(2(g(M) \varepsilon+\delta)+\left\|A^{\prime}(x)\left(I-P_{n}\right) x\right\|+\frac{L}{s !}\left\|\left(I-P_{n}\right) x\right\|^{s}\right)\left\|x_{\omega n}-x^{n}\right\| \\
& +\alpha\left\langle U^{s}\left(x^{n}\right), x^{n}-x_{\omega n}\right\rangle, \quad \forall x \in S_{0}^{G}, x^{n}=P_{n} x .
\end{aligned}
$$

Obviously, this inequality gives us the boundedness of the sequence $\left\{x_{\omega_{n}}\right\}$. Without loss of generality, suppose that $x_{\omega n} \rightarrow x_{1} \in X$, as $n \rightarrow+\infty$ and $\varepsilon, \delta, \alpha \rightarrow 0$. Then $x_{1} \in G$ because $x_{\varepsilon n} \in G$ for every $n$. From (2.3) it follows

$$
\left\langle\left(x^{n}\right)-f_{0}, x^{n}-x_{\omega n}\right\rangle+\alpha\left\langle U^{s}\left(x^{n}\right), x^{n}-x_{\omega n}\right\rangle+2(g(M) \varepsilon+\delta)\left\|x^{n}-x_{\omega n}\right\| \geq 0 .
$$


After passing $n \rightarrow+\infty$ in this inequality, the continuity of $A$ and the weak convergence of the sequence $\left\{x_{\alpha n}\right\}$ give us

$$
\left\langle A(x)-f_{0}, x-x_{1}\right\rangle \geq 0 \forall x \in G .
$$

Now, by the similar way, as in the proof of the Theorem 2.2 we can conclude that the sequence $\left\{x_{\omega n}\right\}$ converges to $x_{0}$. Theorem is proved.

Note that the condition of defining the number $M$ is presented in [13].

\section{REFERENCES}

[1] I. J. Alber and I.P. Ryazantseva, Regularization of nonlinear equations with monotone operators, J. of Comp. Math. and Math. Phys. SSSR 15 (1975) 283-289.

[2] I.J. Alber, On solving nonlinear equation involving monotone operators in Banach spaces, Sibirskii Math. J. 26 (1975) 3-11.

[3] I. J. Alber and I. P. Ryazantseva, On solutions of nonlinear problems involving monotone discontinuous operators, Diffrentsialnye uranenia SSSR 25 (2) (1979) 331-342.

[4] I. J. Alber and A.I. Notik, Geometrical properties of Banach spaces and approximate methods for solving nonlinear operator equations, Dokl. Acad. Nauk SSSR 275 (1984) 1033-1037.

[5] H. W. Engl and C. W. Groetsch, Projection-regularization for linear operator equations of the first kind, in "Special Programs on Inverse problems", Proc. of the Center of Math. Anal., Australia Nat. Univ., 17 (1988) 17-31.

[6] J. L. Lions, Quelques Méthodes de Résolution des Problèmes aux Limites Non Linéaires, Paris, 1969.

[7] O.A. Liskovets, Regularization for problem involving discontinuous monotone operator under arbičrary perturbations, Dokl. Acad. Nauk SSSR 272 (1) (1983) 30-34.

[8] I. P. Ryazantseva, On select of regularizating parameters for equations under monotone perturbations, Izvestia Vyschix Uchebnix Zav., ser. Math. SSSR, N. 8 (1981) 39-43.

[9] I.P. Ryazantseva, Solution of variational inequalities with monotone operators by method of regularization, J. Math. of Comp. and Math. Physics SSSR 23 (2) (1983) 479-483.

[10] I. V. Scrypnik, Nonlinear Elliptic Equations of High Order, Naukova-Dumka, Kiev, 1973.

[11] A. N. Tikhonov and V.la. Arsenin, Solutions of Ill-Posed Problem, Wiley - New York, 1977.

[12] D. Vaclav, Monotone Operators and Applications in Control and Network Theory, Elsevier, Ams. - Oxf. - New-York, 1979.

[13] M. M. Vainberg, Variational Method and Method of Monotone Operators, Nauka, Moscow, 1972.

Tóm tắt. Mục đích của bài báo này là trình bày một sưa đổi tốt hơn cho tiểp cận của Liskovet để giải bài toán không chính quy phi tuyển với toán tử đơn điệu trong không gian Banach phản xạ với toán tử nhiễu bất kỳ. Sự hội tụ cưa nghiệm hiệu chỉnh được xét đến trong mối tương quan vơi xấp xỉ hữu hạn chî̀u cho không gian đó.

Received: April 2, 1998

Institute of Information Technology

National Centre for Sciences and Technology of Vietnam 